\title{
Sleep characteristics in child and adolescent offspring of parents with bipolar disorder: a case control study
}

\author{
Antonin Sebela ${ }^{1,2^{*}}$, Tomas Novak ${ }^{1,4}$, David Kemlink ${ }^{5}$ and Michal Goetz ${ }^{3,6}$
}

\begin{abstract}
Background: Impairment of sleep and circadian rhythm is a typical feature of bipolar disorder (BD). We carried out an exploratory cross-sectional case-control study to extend the knowledge of sleep characteristics in offspring at risk for BD.

Methods: We investigated 42 offspring of bipolar parents (OB) (mean age $12.5 \pm 3.2$ ) and 42 sex and age matched comparison offspring of healthy parents. We administered the Pediatric Sleep Questionnaire, the Morningness/ Eveningness Questionnaire and The General Behavior Inventory Sleep Subscale (GBISS) to assess circadian preference, and to identify sleep impairment symptoms. In addition, the participants completed 14 days of actigraphy to characterise sleep and wake patterns. The current psychopathology profile was assessed using Kiddie Schedule for Affective Disorders and Schizophrenia.

Results: Prevalence of sleep disturbance symptoms was higher among OB than controls (headache after waking up, $17.9 \%$ vs. $2.4 \%, p=0.03$; excessive daytime sleepiness, $38.5 \%$ vs. $10.0 \%, p=0.004$; apparent tiredness at wake-up times, $43.6 \%$ vs. $15.0 \%, p=0.007$ and nightmares, $21.6 \%$ vs. $2.4 \%, p=0.01$ ), but the differences between groups were not significant after adjusting for current psychopathology. OB had higher GBISS total score (parental version, $p<0.001$; self-assessment, $p=0.07$ ) than the controls. $\mathrm{OB}$ had higher preference for eveningness than the controls $(p=0.047)$. According to the actigraphy, OB had longer sleep onset latency $(p=0.048)$ than the controls.
\end{abstract}

Conclusion: Evidence suggests that the offspring of bipolar parents experience sleep disturbance symptoms, which was associated with psychopathology in this study. Prospective longitudinal sleep studies would clarify whether sleep disturbance could be a predictor of mood disorder onset in this high-risk population.

Keywords: Actigraphy, Offspring, Bipolar disorder, Sleep, High-risk, Child, Adolescent

\section{Background}

Bipolar disorder (BD) is a lifelong psychiatric illness that severely impairs quality of life with an estimated lifetime prevalence from 1 to $2 \%$ [1]. The usual age of onset for BD is from late adolescence to early adulthood, and high-risk studies have provided evidence of childhood antecedents [2]. Retrospective studies have shown that approximately $30-60 \%$ of adults with BD reported an illness onset before 20 years of age $[3,4]$ and up to one

\footnotetext{
* Correspondence: antonin.sebela@nudz.cz

'National Institute of Mental Health, Topolova 748, 25067 Klecany, Czech Republic

${ }^{2}$ First Faculty of Medicine, Charles University in Prague, Katerinska 32, 12108

Prague 2, Czech Republic

Full list of author information is available at the end of the article
}

quarter of them recalled that their first mood episode occurred before 13 years of age [5]. However, retrospectively obtained age of onset is both vulnerable to recall bias and focused only on major mood episodes.

Differences in sleep architecture and circadian rhythms are commonly found in adults with BD during manic, depressive and even in euthymic phases of their illness $[6,7]$. Sleep disturbance is also a common prodrome of both mania and depression [8] among patients with bipolar. Recently, Cretu et al. [9] found strong associations between poor sleep quality and shorter time to mood episode recurrence in recovered patients with BD.

The clock-like circadian system and sleep homeostatic self-modulation are regulating processes of the sleep- 
wake cycle [10]. Circadian rhythm disruption is a core feature of BD [11]. Moreover, a recent meta-analysis [12] showed that patients with BD have less stable and more variable circadian activity patterns, even in euthymia, than healthy controls. The period of circadian rhythms is variable among individuals and this variability is marked as chronotype or circadian preference. There are three basic types of chronotype: morning, evening and intermediate [13]. Adult patients with BD mostly have the evening chronotype and this feature is relatively stable $[14,15]$.

Impaired sleep is also an important aspect of paediatric $\mathrm{BD}$. Individuals with $\mathrm{BD}$ may manifest increased sleep onset latency [16] and poorer sleep efficiency [17] than healthy controls. Furthermore, sleep disturbance was found to be an initial symptom of BD in $45 \%$ of children with full-blown paediatric BD in a retrospective study by Faedda [18]. Proper sleep is also important for all children, as strong evidence suggests that impaired sleep in children and adolescents negatively impacts their cognitive and behavioural functioning [19].

$\mathrm{BD}$ is highly heritable, with up to $85 \%$ of variance in risk determined by genetic factors [20] and family history of $\mathrm{BD}$ is the strongest predictor for the development of this illness [21]. Systematic prospective assessment of the offspring of bipolar parents (OB) is therefore the approach of choice to obtain information on the early stages and the development of BD [22]. Evidence from prospective studies of $\mathrm{OB}$ suggests that sleep disturbances are prodromal symptoms of BD [23, 24]. Despite sleep disorders and disturbances being mentioned as initial stages in the developmental model of $\mathrm{BD}$ [25], information about sleep characteristics of $\mathrm{OB}$ is relatively sparse.

Sleep questionnaires and sleep diaries are common subjective methods for evaluating sleep habits and chronotype. Actigraphy and polysomnography are useful objective methods for evaluating sleep characteristics in patients with BD [26]. Each of these methods has its individual advantages and disadvantages. Actigraphic assessment of sleep parameters has been validated in different studies using polysomnography [27], although it has limitations, which should be mentioned. In comparison to polysomnography, actigraphy has a low ability to detect wakefulness during sleep [28]. Further, it does not mirror the polysomnography in estimating sleep onset latency [29].

Polysomnography records multiple electrophysiological parameters at one time and thus can detect many types of sleep disturbances; it is the gold standard for objective assessment of macro-structural sleep parameters and sleep disorders. However, the data are obtained in a sleep laboratory, therefore, the number of nights that can be recorded for each individual subject is limited and thus insufficient for assessment of sleepwake cycle stability. The wrist worn actigraphy has the advantage of being able to evaluate sleep patterns at home, and can be used for longer periods of time. On the other hand, actigraphy measures movement and therefore it is a proxy measure of sleep. To the best of our knowledge, only two studies from the large number of OB studies have specifically evaluated sleep characteristics of offspring with genetic and environmental risk of BD due to illness of parent [30, 31].

The first study dedicated to sleep among $\mathrm{OB}$ was conducted by Jones et al. [30]. The authors used both subjective and objective methods for assessment of sleep patterns among OB. Seven days of actigraphic measurement were used as an objective method. The only statistical difference in sleep between the $\mathrm{OB}$ and the control groups in this study was shorter sleep latency in OB. Furthermore, Levenson et al. [31] used subjective methods for evaluating sleep characteristics of OB. In the $\mathrm{OB}$ group, the authors found higher rates of inadequate sleep, poor sleepers, frequent awakenings and circadian evening preference. But after adjusting for lifetime psychopathology, only parental perception of inadequate sleep of the child remained.

Studying sleep disturbances, circadian patterns and preferences in the offspring at risk for $\mathrm{BD}$ may pave the way for the development of better methods for early diagnostics, early interventions and potentially also for the prevention of $\mathrm{BD}$. To extend the knowledge of sleep characteristics among offspring at risk for $\mathrm{BD}$, we carried out an exploratory cross-sectional case-control study and applied both subjective and objective methods.

\section{Methods}

\section{Participants}

Recruitment of BD families was done using the Czech Bipolar Disorder Case Registry of the National Institute of Mental Health in Klecany, Czech Republic. This register is a database of patients with BD confirmed by the semi-structured psychiatric interview (Schedule for Affective Disorders and Schizophrenia Lifetime version, SADS-L) [32]. We also asked other psychiatric outpatient services across the Czech Republic to inform adults with BD I or II about our survey. We performed preliminary screening interviews with the families that agreed to participate in the project.

The inclusion criteria were as follows: for parent 1) diagnosis of BD-I or BD-II confirmed by SADS-L, 2) no history of psychotic disorder, 3) written, informed consent with study protocol; for offspring 1) age range 6-17; this age range was chosen because we could detect early symptoms of impairment in various domains of development, 2) no history of severe health complications in the mother during pregnancy capable of potential 
impairment of intrauterine development, 3) no history of any chronic physical condition and 4) assent with study protocol.

Families were excluded from our study if they had more than one parent with a mood disorder or if they could not comply with the research protocol. All parents completed the standardised SADS-L post-screening research interviews to confirm the diagnosis of $\mathrm{BD}$.

Community control offspring were recruited through advertisements in schools. Exclusion criteria for the control families included the presence of psychiatric disease in the parents, severe health complications during pregnancy capable of potential impairment of intrauterine development of the offspring, and history of chronic physical condition of offspring. We used preliminary interviews to select families to be included in the control sample. We selected families with offspring that matched the sex and age of the offspring from the BD families.

\section{Offspring assessment}

The semi-structural psychiatric interview Kiddie Schedule for Affective Disorders and Schizophrenia Present and Lifetime version (KSADS-PL) [33] was performed independently on both the offspring and the parents by a trained child psychiatrist. The interviewer was not blind to the status of the parent (control vs. bipolar). DSM-IV diagnoses were confirmed based on consensus review by a board-certified child psychiatrist.

\section{Sleep questionnaires}

We used three questionnaires to assess sleep patterns and disturbances: a) Czech version of the Morningness/ Eveningness Questionnaire (MEQ) to assess circadian preferences [34]. The MEQ is a set of ten questions administered to the child; individual answers have a set value. The morningness/eveningness score was derived from responses to questions about preferred timing of activities such as school tests, bedtime and rising time. The morningness/eveningness score range from 10 to 42 , with higher scores indicating a greater morning tendency. This scale is validated in children of different age groups [35]. b) The Czech version of the Pediatric Sleep Questionnaire (PSQ) was originally developed for investigation of childhood sleep related breathing disorders and prominent symptom complexes, including snoring ( 4 items), breathing problems during sleep (4 items), and daytime sleepiness (4 items). Further, the inattentive/hyperactive behaviour scale (6 items) and other items assessing symptoms of impaired sleep are included in the questionnaire. PSQ is completed by the parent concerning his or her offspring using the yes/no/don't know response format [36]. c) The Czech version of the General Behavior Inventory Sleep Subscale [37] (GBISS) was used to assess sleep disturbances typical for BD.
Both parental and self-assessment versions were used. This subscale comprises seven Likert-type items of the General Behavior Inventory [38] that cover symptoms of initial, middle and terminal insomnia. The GBISS scores range from 0 to 21 , with higher scores indicating more severe disruption of sleep.

\section{Actigraphy}

The objective assessment of sleep and wake patterns was analysed by a wrist worn actigraphic device with a triaxial accelerometer (MotionWatch8, CamNTech, Cambridge, UK, www.camntech.com). The subjects wore the device continuously for two weeks on the nondominant upper extremity. Offspring recorded if and for how long they removed the actigraphic device, with instruction to take it off only when it could get wet. Parents were instructed to fill the sleep diaries including information about wake up and bed times of their offspring. We also used ordinary wake up and bed times from the PSQ to determine the sleep time. We calculated all variables dependent on the precise record of going to bed/waking up using sleep diaries and the most common time of going to bed/waking up as mentioned by parents in the PSQ. Data were sampled in 60-s epochs, stored digitally and analysed using the MotionWare Software v1.1.20 (CamNTech, Cambridge, UK, www.camntech.com). Both standard sleep macrostructure parameters and non-parametric statistics based on a multiple day recording (Non-Parametric Circadian Rhythm Analysis - NPCRA), as implemented in the system, were used for analyses. For the NPCRA, we selected the following variables: Intra-daily variability (IV), which reflects rhythm fragmentation and has a range of 0 to 2 with higher values indicating higher fragmentation. Inter-daily stability (IS) indicates invariability of the daily rhythm between days in the assessed period and has a range of 0 to 1 , where a value of 0 indicates a total lack of rhythm and a value of one indicates a perfectly stable rhythm. Relative amplitude (RA) refers to the quantity of daytime activity. This variable has a range of 0 to 1 with higher values indicating a rhythm with higher amplitude [39]. Actigraphic data analysis was performed by a statistician blind to the status of the offspring (control vs. high-risk).

\section{Statistical analyses}

The demographic and clinical characteristics between the high-risk and control groups were compared using the unpaired t-test, Mann-Whitney U-test, Fisher's exact tests, and Pearson chi-square test as appropriate.

As most sleep related continuous parameters (GBISS, MEQ, actigraphic measures) were non-normally distributed (assessed by Shapiro-Wilk's test), the MannWhitney U-test was used for comparisons between 
groups. Dichotomous variables (PSQ) were compared using Fisher's exact test. Significant differences between groups were consequently adjusted for confounding factors (current psychopathology, psychotropic medication and substance use) using multivariate linear, ordinal or binomial regression analyses to ensure that the differences are attributable to group membership. All statistical procedures were conducted using the STATISTICA software package, version 12.0 (StatSoft, Inc. 2013).

\section{Results}

\section{Demographic variables and the profile of current psychopathology}

We enrolled 34 families with a BD parent (25 cases of BD type I; 9 cases of BD type II) and 33 control families (Table 1). The offspring sample consisted of 42 high-risk offspring and 42 age and sex matched controls (Table 2).

Five cases of bipolar spectrum disorders were found in the OB group (1 case with BD-II, 3 cases with BD-NOS and 1 case with cyclothymia). No cases of bipolar spectrum disorders were found in the control group. Six cases of depressive spectrum disorders were found in the $\mathrm{OB}$ group. No cases of depressive spectrum disorders were found in the control group (Table 2).

\section{Sleep and circadian variables}

For the PSQ, we did not find any significant differences between offspring groups on the main scales (breathing problems during sleep, snoring, daytime sleepiness, inattentive/hyperactive behaviour). We conducted a further analysis on frequency of impaired sleep symptoms (Table 3). Headache after waking up was reported in $17.9 \%$ of the $\mathrm{OB}$ and $2.4 \%$ of controls $(p=0.03)$, excessive daytime sleepiness in $38.5 \%$ of the OB and $10.0 \%$ of controls $(p=0.004)$, apparent tiredness at wake-up times in $43.6 \%$ of the $\mathrm{OB}$ and $15.0 \%$ of controls $(p=0.007)$, and nightmares in $21.6 \%$ of the $\mathrm{OB}$ and $2.4 \%$ of controls $(p=0.01)$. However, after adjusting for potential confounding factors (current psychopathology, medication and substance use), the significant differences between groups on sleep variables were no longer significant: headache after wake-up (adjusted $\mathrm{OR}=2.0$; 95\% CI: 0.15-28.12; $p=0.59$ ), excessive daytime sleepiness $(\mathrm{OR}=2.1 ; 95 \% \mathrm{CI}: 0.49-8.88 ; p=0.32)$, apparent tiredness at wake-up times $(\mathrm{OR}=1.2 ; 95 \%$ CI: 0.30 $4.88 ; p=0.80)$, and nightmares ( $\mathrm{OR}=1.2$; $95 \% \mathrm{CI}: 0.07$ 33.5; $p=0.90$ ). Instead, current psychopathology was associated with sleep disturbances as follows: headache after wake-up (ADHD, OR = 17.5, 95\% CI: 1.4-220.4, $p=0.03$ and anxiety disorders, $\mathrm{OR}=28.8,95 \% \mathrm{CI}: 2.2-$ 378.2, $p=0.01$ ); excessive daytime sleepiness (ADHD, $\mathrm{OR}=6.3,95 \%$ CI: 1.3-31.6, $p=0.02$ and depressive disorders, $\mathrm{OR}=20.4,95 \% \mathrm{CI}: 1.8-230.8, p=0.01)$; apparent tiredness at wake-up times $(\mathrm{ADHD}, \mathrm{OR}=5.8$, 95\% CI: 1.1-31.6, $p=0.04$; anxiety, OR $=9.1,95 \%$ CI: 2.0-41.4, $p=0.001$ and depressive disorders, $\mathrm{OR}=17.2$, 95\%CI: 1.3-234.2, $p=0.03$ ); nightmares (substance use, $\mathrm{OR}=35.9,95 \%$ CI: $1.4-961.3, p=0.03)$.

On the GBISS, parents of the OB reported higher total scores compared to parents of control offspring

Table 1 Parental characteristics

\begin{tabular}{|c|c|c|c|}
\hline & Bipolar families $(N=34)$ & Control families $(N=33)$ & $p$-value \\
\hline \multicolumn{4}{|l|}{ Families } \\
\hline Intact family, N, (\%) & $16(47.1)$ & $25(75.8)$ & $0.02^{\mathrm{a}}$ \\
\hline Father's education, E/H/U, N & $3 / 18 / 13$ & $0 / 13 / 20$ & $0.07^{b}$ \\
\hline Mather's education, E/H/U, N & $2 / 24 / 8$ & $0 / 11 / 22$ & $0.001^{b}$ \\
\hline \multicolumn{4}{|l|}{ Probands } \\
\hline Female/Male & $19 / 15$ & $18 / 15$ & $1.00^{\mathrm{a}}$ \\
\hline \multicolumn{4}{|l|}{ Mood disorders, N, (\%) } \\
\hline BD I & $25(73.5)$ & $\mathrm{n} / \mathrm{a}$ & $\mathrm{n} / \mathrm{a}$ \\
\hline $\mathrm{BD} \|$ & $9(26.5)$ & $\mathrm{n} / \mathrm{a}$ & $\mathrm{n} / \mathrm{a}$ \\
\hline Age at onset of $\mathrm{BD}$, mean $\pm \mathrm{SD}$ & $26.3 \pm 10.7$ & $\mathrm{n} / \mathrm{a}$ & $\mathrm{n} / \mathrm{a}$ \\
\hline No. of $\mathrm{BD}$ episodes, mean \pm SD & $5.4(2.6)$ & $\mathrm{n} / \mathrm{a}$ & $\mathrm{n} / \mathrm{a}$ \\
\hline Suicidal attempt, N, (\%) & $13(38.2)$ & 0 & $<0.001^{\mathrm{a}}$ \\
\hline Psychotic symptoms, N, (\%) & $7(20.6)$ & 0 & $0.01^{\mathrm{a}}$ \\
\hline \multicolumn{4}{|l|}{ Non-mood disorders, N, (\%) } \\
\hline Any anxiety disorder & $4(11.8)$ & $1(3.0)$ & $0.36^{\mathrm{a}}$ \\
\hline Substance abuse $e^{c}$ & $7(20.6)$ & $1(3.0)$ & $0.05^{\mathrm{a}}$ \\
\hline
\end{tabular}

$B D$ bipolar disorder, $E / H / U$ Elementary/High school/University, $N$ number, $n / a$ non-applicable, $S D$ standard deviation

${ }^{\mathrm{a}}$ Fisher's exact test, ${ }^{\mathrm{b}}$ Pearson chi-square test; ${ }^{\mathrm{c}}$ includes alcohol marijuana and amphetamines 
Table 2 Demographic and clinical variables of offspring

\begin{tabular}{|c|c|c|c|}
\hline & $\begin{array}{l}\mathrm{OB} \\
(N=42)\end{array}$ & $\begin{array}{l}\mathrm{CO} \\
(N=42)\end{array}$ & $p$-value \\
\hline \multicolumn{4}{|l|}{ Demographic } \\
\hline Female/Male & $17 / 25$ & $17 / 25$ & $1.00^{\mathrm{a}}$ \\
\hline Age, mean (SD) & $12.5(3.2)$ & $12.4(3.1)$ & $0.7^{\mathrm{b}}$ \\
\hline Psychotropic medication', N, (\%) & $7(16.7)$ & $0(0.0)$ & $0.01^{\mathrm{a}}$ \\
\hline \multicolumn{4}{|l|}{ Current psychopathology, N, \% } \\
\hline Any BD spectrum disorder ${ }^{d}$ & $5(11.9)$ & $0(0.0)$ & $0.06^{\mathrm{a}}$ \\
\hline Any depressive spectrum disorder ${ }^{\mathrm{e}}$ & $6(14.3)$ & $0(0.0)$ & $0.03^{\mathrm{a}}$ \\
\hline Any anxiety disorder ${ }^{f}$ & $13(30.9)$ & $4(9.5)$ & $0.02^{\mathrm{a}}$ \\
\hline Substance use disorder ${ }^{9}$ & $6(14.3)$ & $0(0.0)$ & $0.03^{\mathrm{a}}$ \\
\hline ADHD & $10(23.8)$ & $2(4.8)$ & $0.03^{\mathrm{a}}$ \\
\hline Not currently mentally ill & $17(40.5)$ & $36(85.7)$ & $0.001^{\mathrm{a}}$ \\
\hline
\end{tabular}

$A D H D$ attention-deficit hyperactivity disorder, $B D$ bipolar disorder, $C O$ control offspring, $N$ number, NOS not otherwise specified, $O B$ high-risk offspring, $S D$ standard deviation

${ }^{\mathrm{a}}$ Fisher's exact test, ${ }^{\text {bunpaired t-test }}$

cincluding antidepressants, psychostimulants and antipsychotics

${ }_{\text {including BD II, BD NOS and cyclothymia }}$

eincluding major mood disorder, depression NOS, depressive adjustment disorder and dysthymia

${ }^{f}$ including generalised anxiety disorder, social and separation anxiety

including alcohol, marijuana and amphetamines

Table 3 Parental and self-report of offspring sleep characteristics

\begin{tabular}{|c|c|c|c|}
\hline & $\mathrm{OB}$ & $\mathrm{CO}$ & $p$-value \\
\hline & $(N=42)$ & $(N=42)$ & \\
\hline \multicolumn{4}{|l|}{ PSQ, mean (SD) } \\
\hline Breathing problems during sleep & $0.2(0.4)$ & $0.2(0.6)$ & $0.6^{\mathrm{a}}$ \\
\hline Snoring & $0.1(0.2)$ & $0.2(0.6)$ & $0.1^{\mathrm{a}}$ \\
\hline Daytime sleepiness & $0.8(1.1)$ & $0.5(0.9)$ & $0.2^{\mathrm{a}}$ \\
\hline Inattentive/hyperactivity behaviour & $1.5(1.5)$ & $0.9(1.3)$ & $0.05^{\mathrm{a}}$ \\
\hline \multicolumn{4}{|l|}{ PSQ, specific items, yes, $\%$} \\
\hline Visible daytime sleepiness & 38.5 & 10.0 & $0.004^{b, c}$ \\
\hline Feels tired after sleep & 43.6 & 15.0 & $0.007^{b, c}$ \\
\hline Headaches after wake-up & 17.9 & 2.4 & $0.03^{b, c}$ \\
\hline Nightmares & 21.6 & 2.4 & $0.01^{b, c}$ \\
\hline \multicolumn{4}{|l|}{ GBISS } \\
\hline Parental version, Total score, mean (SD) & $2.3(3.1)$ & $0.4(0.9)$ & $0.001^{a}$ \\
\hline Self-assessment, Total score, mean (SD) & $4.6(4.4)$ & $2.5(2.3)$ & $0.07^{\mathrm{a}}$ \\
\hline \multicolumn{4}{|l|}{ Circadian phase preference } \\
\hline MEQ, mean (SD) & $27.0(6.4)$ & $30.0(4.2)$ & $0.047^{a}$ \\
\hline \multicolumn{4}{|c|}{$\begin{array}{l}\text { CO control offspring, GBISS General Behavior Inventory Sleep Subscale, MEQ } \\
\text { Morningness-Eveningness Questionnaire, OB high-risk offspring, PSQ Pediatric } \\
\text { Sleep Questionnaire, SD standard deviation } \\
\text { a'Mann-Whitney test; } \\
{ }^{b} \text { Fisher's exact test } \\
\text { 'non-significant after adjustment for current psychopathology, medication, } \\
\text { and substance use (ordinal and binomial logistic regression) }\end{array}$} \\
\hline
\end{tabular}

$(2.3 \pm 3.1$ vs. $0.4 \pm 0.9 ; p<0.001$; adjusted $\mathrm{OR}=1.18$; 95\%CI: $1.03-1.46 ; p=0.03)$. Furthermore, in the parental report of the GBISS scale, OB scored significantly higher on the following items: Increased fatigue and increased sleep, regardless of mood and energy levels $(p=0.046)$, increased fatigue and decreased productivity $(p=0.04)$, middle insomnia regardless of mood and energy levels $(p=0.02)$, and depressed mood with sleeponset insomnia $(p=0.04)$. There was no group difference on items regarding decreased need for sleep, elation and increased energy with initial insomnia and terminal insomnia. No significant difference in the total score on the GBISS was found in the child and adolescent self-assessment ( $p=0.07)$. However, in the analysis of separated items, the cases scored significantly higher on the item concerning depressed mood with sleeponset insomnia $(p=0.002)$.

In the MEQ, the observed scores were as follows: $\mathrm{OB}$ (mean $=27.0 ; \mathrm{SD}=6.4$ and range 13-42), controls (mean $=30.0 ; \mathrm{SD}=4.22$ and range 22-39). This difference was statistically significant $(p=0.047)$. (Table 3$)$.

\section{Actigraphy}

Thirty-one OB (completion rate $73.8 \%$ ) and 27 control offspring (completion rate 64.3\%) completed the actigraphic part of the study. We excluded data from those offspring exhibiting administration problems (disagreement with actigraphic assessment: $7 \mathrm{OB}$ and 11 controls, long periods of time without wearing actigraphic device: $3 \mathrm{OB}$ and 4 controls, and vitiation of the actigraph during the assessment: $1 \mathrm{OB}$ and 0 controls). The sleep diary completion rate was $29 \%$ in the OB group ( 9 complete sleep diaries) and 66\% in the control group (18 complete sleep diaries). When comparing actigraphic results, we found longer sleep latency among the $\mathrm{OB}$ compared to the controls, which remained significant even after adjusting for confounding factors $(p=0.048)$. No other intergroup differences on either sleep analysis results nor on the NPCRA were found. Weekdays and weekends were analysed separately for both types of results (Table 4).

\section{Discussion}

This cross-sectional exploratory study evaluated sleep characteristics among offspring of bipolar parent using both subjective and objective methods. Our survey is, to our knowledge, only the second, to evaluate sleep characteristics among the offspring of bipolar parent using such a comprehensive approach. With a total sample size from 58 (objective measurements) to 84 (subjective), we should have been able to detect standardised group difference of even small to medium sizes (Cohen's d of 0.42-0.50) for both the quantitative and qualitative variables if present. 
Table 4 Results of actigraphic measures in offspring

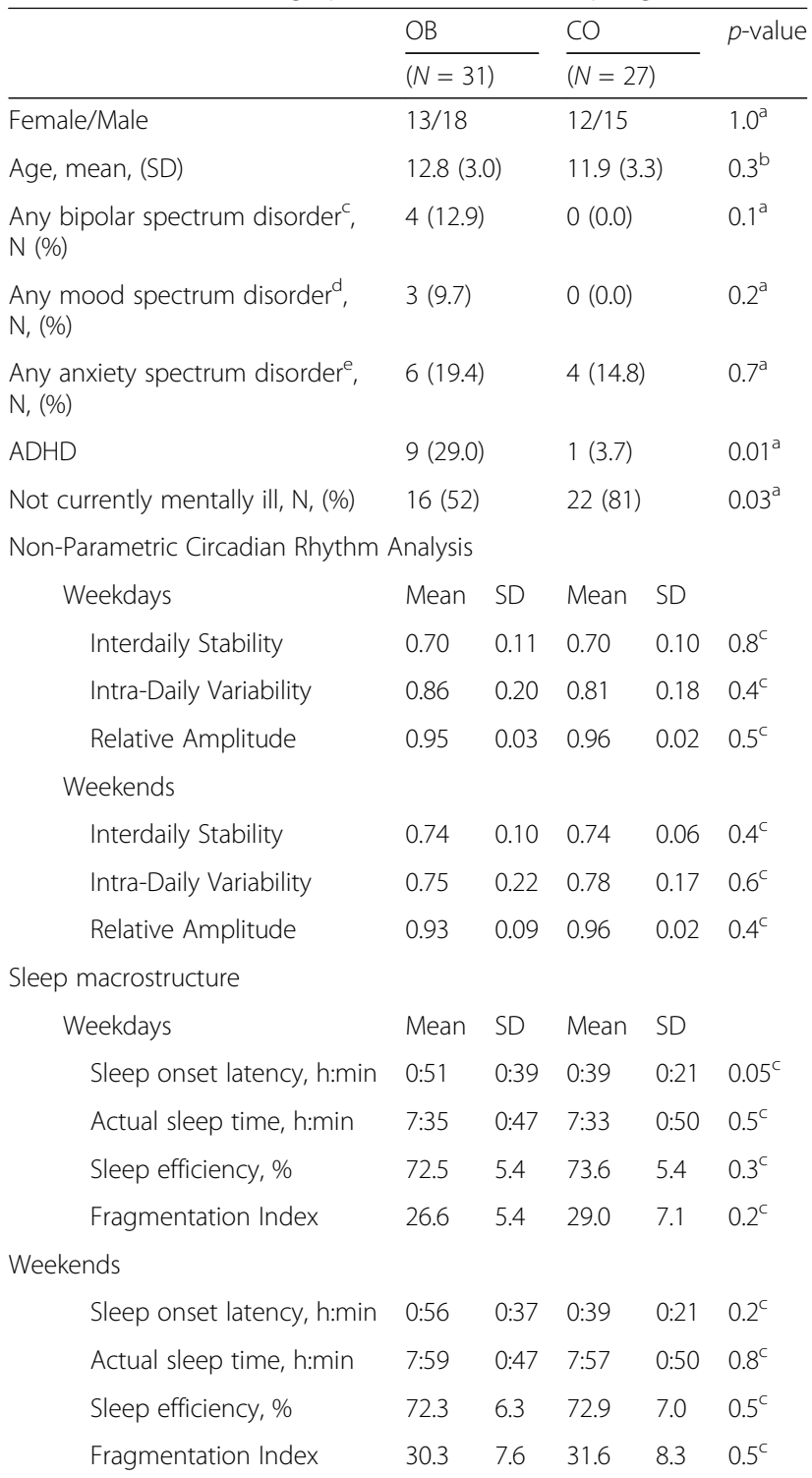

$A D H D$ attention-deficit hyperactivity disorder, $C O$ control offspring, $h$ hour, $\min$ minutes, $N$ number, $O B$ high-risk offspring, $S D$ standard deviation

${ }^{\text {a}}$ Fisher's exact test;

bunpaired t-test;

'Mann-Whitney test

$\mathrm{d}_{\text {including BD II, BD NOS and cyclothymia }}$

eincluding major mood disorder, depression NOS, depressive adjustment disorder and dysthymia

fincluding generalised anxiety disorder, social and separation anxiety

Parents of high-risk offspring reported sleep disturbances in their children more often than the parents of controls. These included: nightmares, feeling of tiredness after sleep, headaches after waking up, excessive daytime sleepiness, increased fatigue, and problems with sleep onset.

However, current psychopathology appeared to be a major confounding factor on impaired sleep quality in our sample (attention-deficit hyperactivity disorder, depressive spectrum disorders, anxiety disorders, and substance use). This is consistent with findings from other studies investigating sleep disturbances in high-risk offspring. Psychiatric medication had no significant effect on the presence of sleep disturbances in our sample. Levenson et al. [32] reported that lifetime psychiatric history had a major effect on the presence of sleep disturbances in high-risk offspring. This limited evidence suggests that impaired sleep found in OB is secondary to psychopathology. Moreover, Jones et al. [31] did not find a significantly higher prevalence of sleep disturbances in their non-symptomatic high-risk sample.

Levenson et al. [32] found a significant circadian preference for going to bed later among high-risk offspring when compared with controls. Moreover, a circadian preference for eveningness has been found in both euthymic paediatric and adult bipolar patients $[15,40]$. The trend in our high-risk sample was similar. Our finding supports the eveningness chronotype preference as a possible trait marker of $\mathrm{BD}$.

Parental reports on OB using the GBISS in our study were approximately the same as the mean total scores reported by the parents of healthy children, and far lower than those reported by the parents of children diagnosed with a disorder from either the bipolar or unipolar spectrum in Meyers and Youngstrom [41]. Children themselves scored higher than parents, but according to our best knowledge, there is no published study using the self-assessment version of the GBISS. Children themselves could be more sensitive to record their sleep disturbances than their parents, but there is a lack of published GBISS data with which to compare our results. However, such discrepancy is consistent with findings from other studies examining parent versus child agreement and disagreement on presence of sleep disturbances [16, 42]. Children are more sensitive to their sleep problems than their caregivers. According to our findings, clinicians should incorporate information from both children and parents in the assessment of sleep impairment among children.

The actigraphic data showed that the $\mathrm{OB}$ group had more difficulties with sleep onset, and that both $\mathrm{OB}$ and control offspring had similar sleep macro-structure, motoric activity and circadian rhythm. On the one hand, our finding of longer sleep latency during the weekdays contrasts with Jones et al. [30], but on the other hand it is consistent with the higher occurrence of sleep-onset insomnia symptoms in our sample. The disparity between our finding and Jones et al. [30] could be caused by the difference in the current psychiatric status of the OB sample - offspring in the sample of Jones were mainly well. We did not find any rest-activity rhythm dysregulation among our OB sample. This could be explained by the timing of the actigraphic assessment. All 
children participating in our survey wore the actigraph during school time. Moreover, they wake up and go to bed regularly under their parents' supervision. Future research should focus on vacation time, when children have a different daily regime than during school time.

The mismatch between subjective parental reports of sleep disturbances in offspring and objective actigraphic measures in our sample is consistent with results from other studies [43, 44]. However, the discrepancy we found in our sample may also be a matter of methodology. It has been shown that symptoms of sleep impairment are not a stable feature in children at risk of BD; rather they wax and wane episodically [44]. Therefore, a 14-day actigraphic assessment may miss this fluctuation. Parents evaluate sleep quality of their offspring in the long-term and therefore may be better able to determine sleep disturbances. Further prospective research, with a longer period of actigraphic assessment, is needed to confirm this hypothesis.

It is well known that the incidence of sleep disturbances as a comorbidity of anxiety and mood disorders is high in the child and adolescent population [45]. However, the causal relationship between sleep disturbances and anxiety and affective disorders is not well described. The question is whether altered sleep quality is a pre-existing trigger of disorder development, or whether it accompanies a psychiatric illness as a part of its manifestation. Understanding this process is important, as disturbed sleep is discussed as a possible early marker of BD [31, 46]. However, the lack of prospective sleep oriented research on offspring at high-risk for BD has prevented us from taking a final stance on this issue.

\section{Limitations}

Several limitations related to our findings must be highlighted. First, the cross-sectional design of our study was less able to detect factors that redound to risk of illness onset in the OB. Second, the completion rate of sleep diaries was low, and consequently, we had to use the approximated bed and wake times from the PSQ in sleep macrostructure analyses. Third, the actigraphic assessment was conducted for two weeks, which might not be a sufficient period to capture impaired circadian rhythm. Fourth, the child psychiatrist who evaluated offspring was not blind to the status of the parent (control vs. bipolar). Lastly, the limited size of our sample did not allow us to separately compare symptomatic highrisk offspring to non-symptomatic high-risk offspring, as well as separate child and adolescent groups.

\section{Conclusions}

Despite the limitations, the present findings have important implications for clinical practice. Children and adolescents at risk for BD appear to manifest symptoms of sleep disturbance, which in this study were strongly associated with current psychopathology. Clinicians taking care of anxious or depressed children and adolescents should incorporate questions aimed at sleep disturbances into their assessment, and should interview their young clients directly. Interventions aimed at both sleep impairment and psychopathology are needed for maximum treatment benefit. Further longitudinal assessment of sleep characteristics is needed to more precisely define the relationship between sleep disturbances and the development of BD.

\section{Abbreviations}

ADHD: Attention-deficit hyperactivity disorderBDBipolar disorder; CO: Control offspring; DSM-IV: Diagnostic and Statistical Manual of Mental Disorders, 4th Edition; GBISS: General Behavior Inventory Sleep Subscale; IS: Inter-daily stability; IV: Intra-daily variability; KSADS-PL: Kiddie Schedule for Affective Disorders and Schizophrenia Present and Lifetime version;

MEQ: Morningness/Eveningness Questionnaire; NPCRA: Non-Parametric Circadian Rhythm Analysis; OB: Offspring at the genetic and environmental risk of bipolar disorder; PSQ: Pediatric Sleep Questionnaire; RA: Relative amplitude; SADS-L: Schedule for Affective Disorders and Schizophrenia Lifetime version; UK: United Kingdom

\section{Acknowledgments}

We thank Adam Whitley for the language editing. We thank Anne Duffy and Paul Grof for their inspirational suggestions. We also warmly thank our research families.

\section{Funding}

This study was funded by the project Nr. LO1611 with financial support from the Ministry of Education, and by MH CZ - DRO, Motol University Hospital, Prague, Czech Republic 00064203, and by the Ministry of Health of the Czech Republic, grant nr. 16-31380A and 17-32478A, and by the Charles University, project GA UK no. 476417. MG served as a speaker for Eli Lilly Czech Republic in the last three years. The other authors of this paper do not have any commercial associations that might pose a conflict of interest in connection with this manuscript.

\section{Availability of data and materials}

The authors confirm that, for approved reasons, some access restrictions apply to the data underlying the findings. The data set contains identifying participant information, which is not suitable for public deposition. Data might be available upon request by contacting the corresponding author; however, the request must comply with confidentiality and ethics rules of the ethics committee of our institution.

\section{Authors' contributions}

AS, MG, and TN contributed to the study design, AS and MG were responsible for data acquisition, TN and DK conducted the data analysis, AS and DK interpreted the data, and AS, TN, MG, and DK helped with the manuscript preparation. All authors read and approved the final manuscript.

\section{Competing interests}

The authors declare that they have no competing interests.

\section{Consent for publication}

Not applicable.

\section{Ethics approval and consent to participate}

The study was approved by the ethics committee of 2nd Faculty of Medicine, Charles University in Prague and the ethics committee of National Institute of Mental Health, Klecany. Written, informed consent from parents and assent from offspring regarding the study protocol were obtained. 


\section{Publisher's Note}

Springer Nature remains neutral with regard to jurisdictional claims in published maps and institutional affiliations.

\section{Author details \\ ${ }^{1}$ National Institute of Mental Health, Topolova 748, 25067 Klecany, Czech Republic. ${ }^{2}$ First Faculty of Medicine, Charles University in Prague, Katerinska 32, 12108 Prague 2, Czech Republic. ${ }^{3}$ Second Faculty of Medicine, Charles University in Prague, V Uvalu 84, 515006 Prague, Czech Republic. ${ }^{4}$ Third Faculty of Medicine, Charles University in Prague, Ruska 87, 1010000 Prague, Czech Republic. ${ }^{5}$ Department of Neurology, General Teaching Hospital and First Faculty of Medicine, Charles University in Prague, Katerinska 30, 12821 Prague 2, Czech Republic. 'Department of Child and Adolescent Psychiatry, Motol University Hospital, V Uvalu 84, 515006 Prague, Czech Republic.}

Received: 14 August 2016 Accepted: 17 May 2017

Published online: 26 May 2017

\section{References}

1. Merikangas KR, Jin R, He JP, et al. Prevalence and correlates of bipolar spectrum disorder in the world mental health survey initiative. Arch Gen Psychiatry. 2011:68:241-51.

2. Holtzman JN, Miller S, Hooshmand F, et al. Childhood-compared to adolescent-onset bipolar disorder has more statistically significant clinical correlates. J Affect Disord. 2015;179:114-20.

3. Egeland JA, Hostetter AM, Pauls DL, Sussex JN. Prodromal symptoms before onset of manic-depressive disorder suggested by first hospital admission histories. J Am Acad Child Adolesc Psychiatry. 2000;39:1245-52.

4. Morselli PL, Elgie R. GAMIAN-Europe*/BEAM survey I - global analysis of a patient questionnaire circulated to 3450 members of 12 European advocacy groups operating in the field of mood disorders. Bipolar Disord. 2003;5:265-78.

5. Perlis RH, Miyahara S, Marangell LB, et al. Long-Term implications of early onset in bipolar disorder: data from the first 1000 participants in the systematic treatment enhancement program for bipolar disorder (STEP-BD). Biol Psychiatry. 2004:55:875-81.

6. Gruber J, Miklowitz DJ, Harvey AG, et al. Sleep matters: sleep functioning and course of illness in bipolar disorder. J Affect Disord. 2011;134:416-20.

7. Sylvia LG, Dupuy JM, Ostacher MJ, et al. Sleep disturbance in euthymic bipolar patients. J Psychopharmacol. 2012;26(8):1108-12.

8. Jackson A, Cavanagh J, Scott J. A systematic review of manic and depressive prodromes. J Affect Disord. 2003;74:209-17.

9. Cretu JB, Culver JL, Goffin KC, Shah S, Ketter TA. Sleep, residual mood symptoms, and time to relapse in recovered patients with bipolar disorder. J Affect Disord. 2016;19:162-6.

10. Borbély AA. A two process model of sleep regulation. Hum Neurobiol. 1982; 1(3):195-204.

11. Gonzalez R. The relationship between bipolar disorder and biological rhythms. J Clin Psychiatry. 2014;75(4):e323-31.

12. Melo MC, Abreu RL, Linhares Neto VB, de Bruin PF, de Bruin VM. Chronotype and circadian rhythm in bipolar disorder: a systematic review. Sleep Med Rev. 2016. doi: 10.1016/j.smrv.2016.06.007.

13. Roenneberg T, Merrow M. Entrainment of the human circadian clock. Cold Spring Harb Symp Quant Biol. 2007;72:293-9.

14. Wood J, Birmaher B, Axelson D, et al. Replicable differences in preferred circadian phase between bipolar disorder patients and control individuals. Psychiatry Res. 2009;166:201-9.

15. Ahn YM, Chang J, Joo YH, Kim SC, Lee KY, Kim YS. Chronotype distribution in bipolar I disorder and schizophrenia in a Korean sample. Bipolar Disord. 2008;10(2):271-5

16. Lofthouse N, Fristad M, Splaingard M, Kelleher K. Parent and child reports of sleep problems associated with early-onset bipolar spectrum disorders. J Fam Psychol. 2007;21(1):114-23.

17. Mehl RC, O'Brien LM, Jones JH, Dreisbach JK, Mervis CB, Gozal D. Correlates of sleep and pediatric bipolar disorder. Sleep. 2006;29:193-7.

18. Faedda GL, Baldessarini RJ, Glovinsky IP, Austin NB. Pediatric bipolar disorder: phenomenology and course of illness. Bipolar Disord. 2004;6(4):305-13.

19. Fallone G, Owens JA, Deane J. Sleepiness in children and adolescents: clinical implications. Sleep Med Rev. 2002;6(4):287-306.

20. Smoller JW, Finn CT. Family, twin, and adoption studies of bipolar disorder Am J Med Genet C Semin Med Genet. 2003;123C:48-58.
21. Lapalme M, Hodgins S, LaRoche C. Children of parents with bipolar disorder: a metaanalysis of risk for mental disorders. Can J Psychiatr. 1997;42:623-31.

22. Duffy A, Doucette $S$, Lewitzka U, Alda M, Hajek T, Grof P. Findings from bipolar offspring studies: methodology matters. Early Interv Psychiatry. 2011:5:181-91.

23. Duffy A, Horrocks J, Doucette S, Keown-Stoneman C, McCloskey S, Grof P. The developmental trajectory of bipolar disorder. Br J Psychiatry. 2014;204:122-8.

24. Egeland JA, Endicott J, Hostetter AM, Allen CR, Pauls DL, Shaw JA. A 16-year prospective study of prodromal features prior to BPI onset in well Amish children. J Affect Disord. 2012;142:186-92.

25. Duffy A. Toward a comprehensive clinical staging model for bipolar disorder: integrating the evidence. Can J Psychiatr. 2014;59:659-66.

26. Kaplan KA, Talbot LS, Gruber J, Harvey AG. Evaluating sleep in bipolar disorder: comparison between actigraphy, polysomnography, and sleep diary. Bipolar Disord. 2012;14:870-9.

27. Martin JL, Hakim AD. Wrist actigraphy. Chest. 2011:139:1514-27.

28. Sitnick SL, Goodlin-Jones BL, Anders TF. The use of actigraphy to study sleep disorders in preschoolers: some concerns about detection of nighttime awakenings. Sleep. 2008;31(3):395-401.

29. Lichstein KL, Stone KC, Donaldson J, et al. Actigraphy validation with insomnia. Sleep. 2006:29(2):232-9.

30. Jones SH, Tai S, Evershed K, Knowles R, Bentall R. Early detection of bipolar disorder: a pilot familial high-risk study of parents with bipolar disorder and their adolescent children. Bipolar Disord. 2006;8:362-72.

31. Levenson JC, Axelson DA, Merranko J, et al. Differences in sleep disturbances among offspring of parents with and without bipolar disorder: association with conversion to bipolar disorder. Bipolar Disord. 2015:17:836-48.

32. Endicott J, Spitzer RL. A diagnostic interview: the schedule for affective disorders and schizophrenia. Arch Gen Psychiatry. 1978;35:837-44.

33. Kaufman J, Birmaher B, Brent D, et al. Schedule for affective disorders and schizophrenia for school-age children-present and lifetime version (K-SADSPL): initial reliability and validity. J Am Acad Child Adolesc Psychiatry. 1997; 36:980-8.

34. Horne JA, Ostberg O. A self-assessment questionnaire to determine morningness-eveningness in human circadian rhythms. Int J Chronobiol. 1976:4:97-110.

35. Carskadon MA, Vieira C, Acebo C. Association between puberty and delayed phase preference. Sleep. 1993;16:258-62.

36. Chervin RD, Hedger K, Dillon JE, Pituch KJ. Pediatric sleep questionnaire (PSQ): validity and reliability of scales for sleep-disordered breathing, snoring, sleepiness, and behavioral problems. Sleep Med. 2000;1:21-32.

37. Meyers OI, Youngstrom EA. A Parent General Behavior Inventory subscale to measure sleep disturbance in pediatric bipolar disorder. J Clin Psychiatry. 2008;69:840-3.

38. Youngstrom EA, Findling RL, Danielson CK, Calabrese JR. Discriminative validity of parent report of hypomanic and depressive symptoms on the General Behavior Inventory. Psychol Assess. 2001:13:267-76.

39. Kim KL, Weissman AB, Puzia ME, et al. Circadian phase preference in pediatric bipolar disorder. J Clin Med. 2014;3:255-66.

40. Owens JA, Spirito A, McGuinn M, Nobile C. Sleep habits and sleep disturbance in elementary school-aged children. J Dev Behav Pediatr. 2000: 21(1):27-36

41. Van Someren EJ, Swaab DF, Colenda CC, Cohen W, McCall W, Rosenquist PB. Bright light therapy: improved sensitivity to its effects on rest-activity rhythms in Alzheimer patients by application of nonparametric methods. Chronobiol Int. 1999:16:505-18.

42. Shaw JA, Egeland JA, Endicott J, Allen CR, Hostetter AM. A 10-year prospective study of prodromal patterns for bipolar disorder among Amish youth. J Am Acad Child Adolesc Psychiatry. 2005;44:1104-11.

43. Cortese S, Faraone SV, Konofal E, Lecendreux M. Sleep in children with attention-deficit/hyperactivity disorder: meta-analysis of subjective and objective studies. Psychophysiology. 1997:34(6):694-702.

44. Sadeh A, Raviv A, Gruber R. Sleep patterns and sleep disruptions in schoolage children. Dev Psychol. 2000;36(3):291-301.

45. Cousins JC, Whalen DJ, Dahl RE, et al. The bidirectional association between daytime affect and nighttime sleep in youth with anxiety and depression. J Pediatr Psychol. 2011;36:969-79.

46. Duffy A. The early natural history of bipolar disorder: what we have learned from longitudinal high-risk research. Can J Psychiatr. 2010;55:477-85. 